\title{
Influence of floodplain and riparian vegetation in the conveyance and structure of turbulent flow in compound channels
}

\author{
João N. Fernandes ${ }^{1}$, João B. Leal ${ }^{2}$, and António H. Cardoso ${ }^{3}$ \\ ${ }^{1}$ LNEC, Lisbon, Portugal \\ ${ }^{2}$ Faculty of Engineering and Science, Universitetet i Agder, Jon Lilletunsvei 9, 4898 Grimstad, Norway \\ ${ }^{3}$ CERIS and DECivil, Instituto Superior Técnico, Universidade de Lisboa, Portugal
}

\begin{abstract}
The present study aims at understanding the changes in the channel conveyance and in the turbulent flow structure due to the presence of both submerged vegetation in the floodplains and riparian vegetation. An experimental campaign was carried out comprising uniform compound channels flows (i) without any kind of vegetation, (ii) with synthetic grass in the floodplains, (iii) with synthetic grass in the floodplains and rods in the interface between main channel and the floodplain and (iv) with synthetic grass in the floodplains and artificial shrubs in the interface between main channel and the floodplain. For comparison, the water depth in all flow cases was kept constant. Accurate acoustic Doppler velocimetry was used to evaluate the $3 \mathrm{~d}$ velocity field and the turbulence structures characteristics.
\end{abstract}

\section{Introduction}

In compound channels, the velocity gradient between the main channel and the floodplain flows leads to a flow structure that is rather more complex than in common single channels (e.g. [1]). Pioneering works on the flow structure in compound channels identified the momentum transfer between the main channel and the floodplain flows as a major difference. In such channels, vortices with vertical and horizontal axis interact and the flow discharge is not as easily predicted as for single channels.

Commonly, floodplains support the existence of vegetation in the whole floodplain and close to the interface between the main channel and the floodplain i.e. riparian vegetation. In both cases, whenever the floodplain is inundated, the vegetation interacts with the flow, slowing it in the vegetated areas, deflecting it to non-vegetated areas and reducing the overall channel conveyance.

The influence of the vegetation in the flow is related to the vegetation elements type, namely, if they are rigid or flexible, submerged or partly submerged and with or without foliage. In the absence of foliage or flexible vegetation, riparian vegetation can be interpreted as vertical rods. The impact of those obstacles in the flow are characterized by the drag force related to friction between the fluid and the surfaces of the rod and with the turbulent eddy motions due to the pressure drop in the wake. The drag force of a group of rods depends on their space density. The spacing ratio, $L / D$, where $L$ is the distance between two consecutive rods with diameter $\mathrm{D}$, may be used to describe different flow structures. 
In this paper, four uniform flows in a symmetric trapezoidal compound channel are characterized. Flow cases comprise compound channel flows (i) without any sort of vegetation, (ii) with vegetated floodplains, (iii) with vegetated floodplains and rods as riparian vegetation and (iv) with vegetated floodplains and shrubs as riparian vegetation.

The influence of the floodplain vegetation and of the riparian vegetation is analysed namely in what concerns to the channel conveyance, the cross sectional distribution of the streamwise velocity, the turbulence characteristics, the coherent structures and the secondary currents. Additionally, for the riparian vegetation, the effects of foliage are also assessed.

\section{Experimental campaign}

The experiments were carried out in a $10 \mathrm{~m}$ long and $2 \mathrm{~m}$ wide compound channel. The symmetrical cross section is composed of a trapezoidal main channel (bank full height $h_{b}=0.1 \mathrm{~m}$, bottom width $b_{m c}=0.4 \mathrm{~m}$, bank full width $B_{m c}=0.6 \mathrm{~m}$, with a side slope of $45^{\circ}$ ) and two lateral rectangular floodplains (width $B_{f p}=0.7 \mathrm{~m}$ each). The channel is made of polished concrete and has a bottom slope of $0.0011 \mathrm{~m} / \mathrm{m}$. A schematic representation of the compound channel is presented in Fig. 1(a). The experimental procedure and more details on the water supply system can be consulted in [2].

The vegetated floodplains were obtained by covering their bottoms with a $5 \mathrm{~mm}$ high synthetic grass. For the polished concrete, $n=0.0092 \mathrm{~m}^{-1 / 3} \mathrm{~s}$ and $k_{s}=0.15 \mathrm{~mm}$ and for the synthetic grass, $n=0.0172 \mathrm{~m}^{-1 / 3} \mathrm{~s}$ and $k_{s}=6.8 \mathrm{~mm}$.
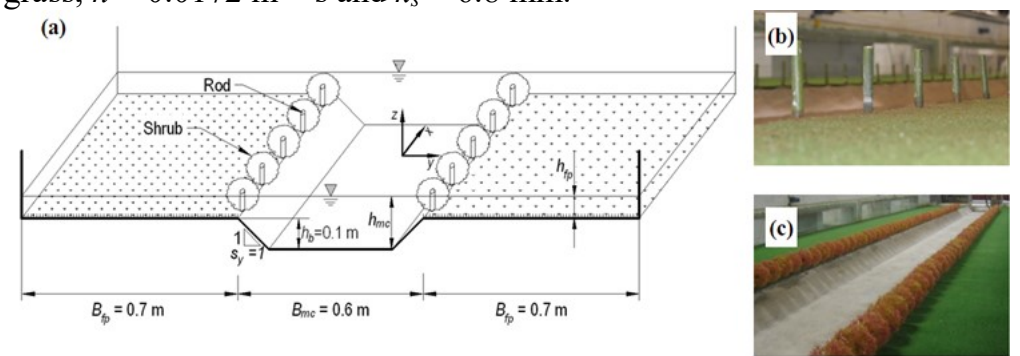

Fig. 1. (a) Schematic representation of the compound channel and (b) photograph of the compound channel with vegetated floodplains and rods at the interface, (c) shrubs at the interface.

For the simulation of the riparian vegetation, two different types of vertical elements were considered in order to mimic the presence of trees and shrubs. The trees consisted of $6 \mathrm{~mm}$ diameter and $45 \mathrm{~mm}$ high vertical elements of rigid, partly-submerged rods without foliage (Fig. 1(b)). The shrubs differ from the trees as the rods present a $90 \mathrm{~mm}$ diameter crown with a spherical shape located radially around the centre on top of the vertical rods (Fig. 1(c)). Despite the flexibility of the branches, the shape of the foliage remained practically unchanged during the tests. The shrubs have approximately 6 to 8 branches. $\mathrm{cm}^{-3}$, corresponding to a high degree of ramification.

There are few studies concerning the effect of geometry and arrangement of the riparian vegetation. [3] reported typical values of spacing ratios, $L / D$, between 8 and 16 . For three rivers in Japan, [4] found values of $L / D$ around 12 to 16. [5] presented typical values of $L / D$ between 12 and 18 in river Thames and approximately equal to 10 in river Rhone. According to [6], tree cultivation spacing $L / D$ equal to 10 are normally recommended. In the present experimental campaign, the spacing between vertical elements is equal to $90 \mathrm{~mm}(L / D=15)$ leading to a situation where the crowns of consecutive elements with foliage touch each other but do not overlap in the longitudinal direction.

Fig. 1(a) presents the reference frame. The longitudinal direction is defined by the $x$-axis, starting at the inlet cross section where $x=0$. The $y$-axis refers to the lateral distance to the 
centre of the main channel and the vertical direction is defined locally by the $z$-axis. After verifying the symmetry of the flow conditions, only half of the cross section was investigated. In the half cross section, the measuring mesh comprised 22 measuring verticals, 3 measuring points per vertical in the floodplain (between $0.4 h_{f p}$ and $0.8 h_{f p}$ ) and 7 in the main channel (between $0.10 h_{m c}$ and $h_{b}+0.8 h_{f p}$ ). Water surface levels were surveyed with a point gauge (accuracy of $\pm 0.3 \mathrm{~mm}$ ) in 9 cross sections at 12 lateral positions per cross section.

The measurements were made at $x=7.5 \mathrm{~m}$, where the boundary layer and the mixing layer are fully developed. This cross section is half way between two consecutive artificial vegetation elements. Additional measurements were conducted in cross sections $x=7.455$ $\mathrm{m}, 7.470 \mathrm{~m}, 7.485 \mathrm{~m}, 7.515 \mathrm{~m}$ and $7.530 \mathrm{~m}$.

Velocity measurements were performed with a $3 \mathrm{~d}$ side looking Vectrino ADV. The sampling rate is $100 \mathrm{~Hz}$ and the acquisition time is $3 \mathrm{~min}$ at each measurement position. For the calculation of the power spectra density, the acquisition time was increase to $10 \mathrm{~min}$.

\section{Hydraulic conditions}

Four uniform flow tests comprising experiments with and without vegetation in the floodplain and in the interface edge of the main channel and floodplain were run. Uniformity was achieved through an iterative procedure in order to get longitudinal constant flow depths and subsection flow discharge distributions. The most important tested variables are listed in Table 1, where $Q m c$ and $Q f p$ stand for the flow discharge in the main channel and in the floodplains, respectively. For dimension similarity, the relative depth, $h_{r}$, defined as the ratio between the floodplain and the main channel flow depths, was kept approximately constant and equal to 0.3 . Each flow case is referenced by " $S$ " or " $R$ " for smooth (polished concrete) or rough (synthetic grass) floodplains, respectively. Additional "R" or "S" was use for referring flow cases with rods or shrubs, respectively.

Table 1 also includes the Froude and the Reynolds numbers in each subsection $i$ (subscript $m c$ and $f p$ stand for the main channel and the floodplain, respectively).

Table 1. Conditions of the flow cases.

\begin{tabular}{|c|c|c|c|c|c|c|c|}
\hline Flow case & $h_{m c}(\mathrm{~m})$ & $Q_{m c}\left(1 . \mathrm{s}^{-1}\right)$ & $Q_{f p}\left(1 . \mathrm{s}^{-1}\right)$ & $\operatorname{Re}_{m c}\left(\mathrm{x} 10^{5}\right)$ & $\operatorname{Re}_{f p}\left(\mathrm{x} 10^{5}\right)$ & $F r_{m c}$ & $F r_{f p}$ \\
\hline $\mathrm{S}$ & 0.140 & 54.2 & 26.4 & 3.17 & 0.66 & 0.70 & 0.76 \\
\hline $\mathrm{R}$ & 0.145 & 42.3 & 16.6 & 2.45 & 0.44 & 0.52 & 0.41 \\
\hline $\mathrm{RR}$ & 0.142 & 41.3 & 14.9 & 3.05 & 0.40 & 0.15 & 0.13 \\
\hline $\mathrm{RS}$ & 0.143 & 39.3 & 11.7 & 2.90 & 0.32 & 0.14 & 0.10 \\
\hline
\end{tabular}

The values of $\operatorname{Re}_{i}$ are markedly higher in the main channel than in the floodplain, but for both subsections are high enough to neglect viscosity effects. The flow is subcritical $(\mathrm{Fr}<1)$ in all tests.

\section{Results and discussion}

\subsection{Channel conveyance}

For the same relative depth, the flow discharge is reduced with the presence of any type of vegetation. Comparing the flow discharge between the compound channel with and without vegetated floodplains, a reduction on the total discharge equal to $27 \%$ is observed when the floodplains are covered with synthetic grass. This reduction is mainly allocated to the decrease of the flow discharge in the floodplain due to the higher roughness of the vegetated 
floodplains. The stronger interaction between the flows in the main channel and in the vegetated floodplains leads to a reduction of the main channel flow discharge of $22 \%$.

The decrease in the compound channel flow discharge due to the riparian vegetation is $5 \%$, for rods, and $13 \%$, for shrubs, in comparison to flow cases with vegetated floodplains without riparian vegetation. These decreases are mainly due to the reduction in the floodplain discharges.

\subsection{Time-averaged streamwise velocity distribution}

The cross sectional distribution of the time-averaged streamwise velocity, $\bar{u}$, scaled by the averaged cross section streamwise velocity, $U_{\text {ave }}$ are shown in Fig. 2 for all flow cases.
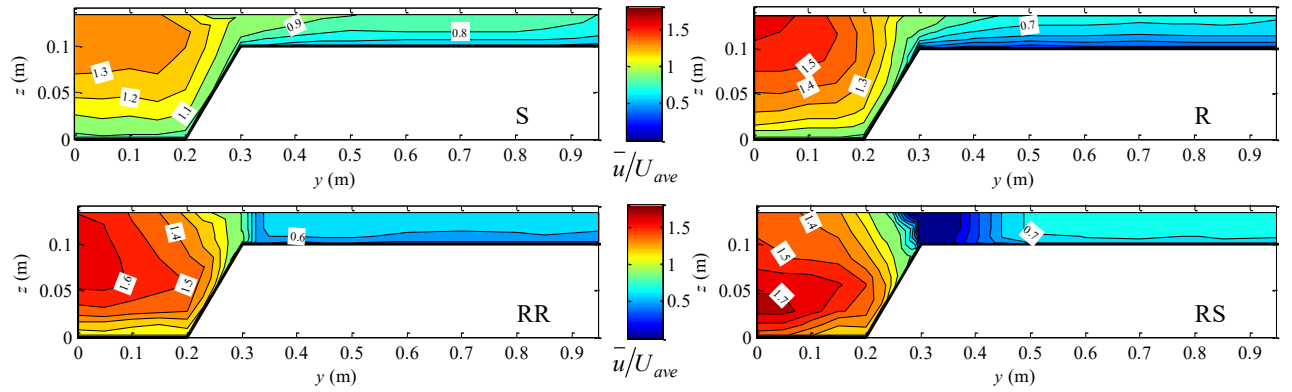

Fig. 2. Cross sectional distribution of the streamwise velocity, $\bar{u}$, scaled by the cross section averaged streamwise velocity, $U_{\text {ave }}$.

The cross sectional distribution of the scaled time-averaged streamwise velocity for smooth and rough (synthetic grass) floodplains cases without riparian vegetation are rather similar (Fig. 2). In both cases, the depth-averaged streamwise velocity can be described by a monotonic function decreasing from the centre of the channel into the end of the floodplain. The lateral gradient of the time-averaged streamwise velocity is mainly observed near the interface where the existence of an inflection point (Rayleigh's criterion) suggests the generation of horizontal vortices.

The depth-averaged velocity increases in the main channel for vegetated floodplains. An important noticeable difference between compound channels with and without vegetation in the floodplains consists in the increase of the velocity lateral gradient between main channel and floodplain flows for the case of vegetated floodplains as a consequence of the increase of the flow resistance in the latter case.

Near the interface between the main channel and the floodplains, the patterns of the streamwise velocity distributions are deeply influenced by both rods and shrubs (RR and RS in Fig. 2). The depth-averaged streamwise velocity with rods in the interface does not follow a monotonic function instead it presents a flat step in the floodplain. With shrubs at the interface, the link between the flows in the main channel and floodplain seems to be broken and a valley in the depth-averaged streamwise velocity is observed with streamwise velocities in the interface close to zero. Nevertheless, in the regions far from the interface the depthaveraged velocity is not much influenced by the riparian vegetation. In those regions, the velocity difference in the main channel and in the floodplain is roughly the same for the 3 flow cases with synthetic grass in the floodplains.

For flow cases with riparian vegetation the streamwise velocity pattern in the main channel is marked by a bulge of the isovels from the interface towards the center of the main channel (see RR and RS in Fig. 2). This effect is also observed in the cases without riparian vegetation ( $\mathrm{S}$ and $\mathrm{R}$ ) but not as clear as for flow cases with riparian vegetation. In these latter, 
similar to the findings of [7] for rods in the interface, the maximum velocity region is not observed near the surface the main channel flow, but moves towards its bottom. This effect is particularly noticeable in the case of shrubs, being their influence felt in whole main channel flow where velocity isovels present a concavity upwards. This can even be better seen in the planview of the depth-averaged streamwise velocity (Fig. 3) for rods and shrubs cases. It is clear that the riparian vegetation originates turbulent structures that detach from the vegetation elements and perturb the flow both in the main channel and flood plain. This effect is neater for the shrubs (RS) case where the perturbations extend more both into the main channel and the floodplain. Although several authors (e.g. [8]) have put focus on the existing secondary currents that are enhanced by the presence of the riparian vegetation, the results in Fig. 3 point out for a much more important role of the vertical oriented turbulent structures originated by the interaction of the flow with vegetation elements.
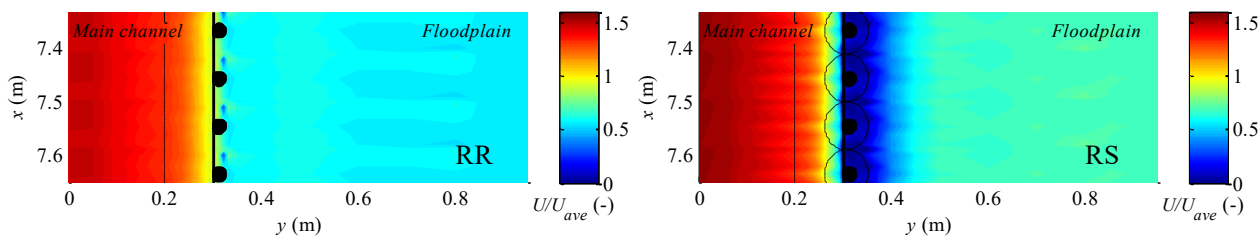

Fig. 3. Planview of the depth-averaged streamwise velocity around the riparian vegetation element.

\subsection{Lateral shear stress}

The cross sectional distribution of the scaled lateral shear stresses, $-\overline{u^{\prime} v^{\prime}} / u_{*}^{2}$, is shown in Fig. 4 for all flow cases and their depth-averaged values $\int-\overline{u^{\prime} v^{\prime}} / u_{*}^{2} d z$ are presented in Fig. 5.
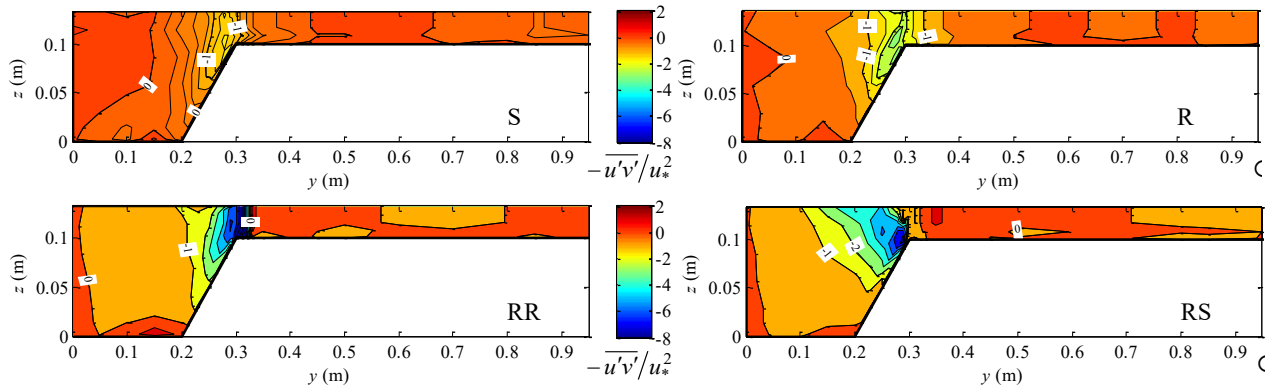

Fig. 4. Cross sectional distribution of the lateral shear stress $-\overline{u^{\prime} v^{\prime}} / u_{*}^{2}$.

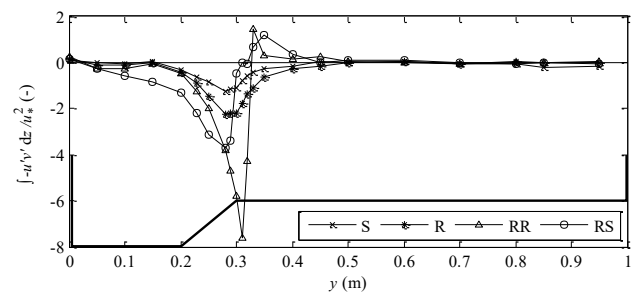

Fig. 5. Depth-averaged lateral shear stress $\int-\overline{u^{\prime} v^{\prime}} / u_{*}^{2} d z$.

For all flow cases, the highest shear region is located near the interface between the main channel and the floodplain. Far from that location interface $-\overline{u^{\prime} v^{\prime}} / u_{*}^{2}$ becomes negligible. When compared with smooth floodplains (S), the presence of synthetic grass in the floodplain (R) does not appreciably change the cross sectional pattern of the shear layer but it increases 
the magnitude of lateral shear stress close to the interface. In the absence of riparian vegetation, in the main channel, a significant vertical and lateral spreading of the shear region towards the main channel side slope is observed. This shear layer also extends laterally towards the floodplain.

The distribution of the lateral shear stresses is deeply affected by the presence of the riparian vegetation. Two common features are observed for both rods (RR) and shrubs (RS): (i) the lateral shear stress in the interface increases (ii) the wake-structure generated by the vegetation elements originates positive lateral shear stress on the floodplain side, since the flow velocity in the latter is higher than the one in the near region of the vegetation elements.

For rods (RR), the spreading of the shear region towards the main channel is similar to what happens without the riparian vegetation. Different patterns are observed for shrubs (RS) in which the spreading is stronger towards the main channel as was mentioned before.

With riparian vegetation two different peaks are observed: one with a negative sign between the vegetation element and the main channel flow and another one positive, between the vegetation element and the floodplain. At some point between the two, the lateral shear stress is zero suggesting that the vegetation suppresses the momentum exchange between the main channel and the floodplain flows. This means that the lateral shear stress is not due to the interaction between main channel and floodplain flows but rather a consequence of the interaction of wake-structure generated by riparian vegetation and the flow in each of those sub-sections. This can be better observed by looking to the planview of the depth-averaged lateral shear stress presented in Fig. 6. Both, the main channel and the floodplain show perturbations arising from the vegetation elements, which are due to the wake-structure that is created on both sides of those elements. The influence of the shrubs (RS) extends more laterally into those two sub-sections, although the rods (RR) present higher local absolute values of the shear stress, as also seen in Fig. 5. It should be pointed out that in both cases the mixing layer width is affected by the presence of riparian vegetation. In the case of shrubs, it is attenuated by the presence of the flexible leafs.
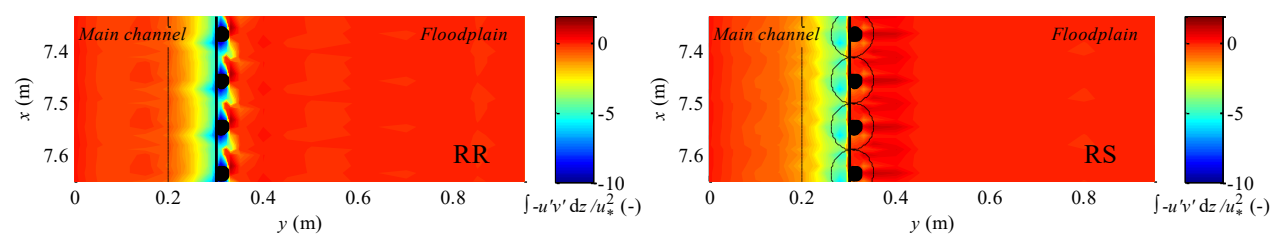

Fig. 6. Planview of the depth-averaged lateral shear stress $\int-\overline{u^{\prime} v^{\prime}} / u_{*}^{2} d z$ around the riparian vegetation.

\subsection{Coherent 2D structures}

The coherent structures in the mixing layer between the main channel and the floodplain flows may be recognized by the power spectra density and by the auto-correlation between the velocity fluctuations over a given time span $\Delta t$, given by:

$$
R_{x x}=\frac{\overline{u^{\prime}(t) u^{\prime}(t+\Delta t)}}{\overline{u^{\prime}(t) u^{\prime}(t)}}
$$

$R_{y y}$ may be obtained by replacing $u$ ' by $v^{\prime}$ in the previous equation.

Fig. 7 presents these functions for a point located in the interface, at a fixed position $x=$ $7.5 \mathrm{~m}$ and $z=h b+0.4 h f$. Power spectra density is presented as a function of the wavenumber $k$ by $S_{x x}$ and $S_{y y}$ for the streamwise and spanwise velocity fluctuations, respectively.

There are clear differences between the autocorrelation for the four flow cases. The case with smooth floodplain feature an almost insignificant modulation of the autocorrelation functions of the fluctuation velocities suggesting the absence of large coherent structures. 
(a)
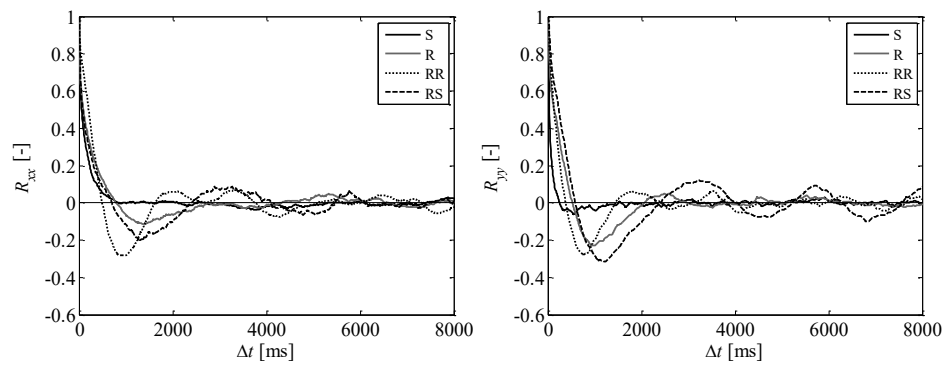

(b)
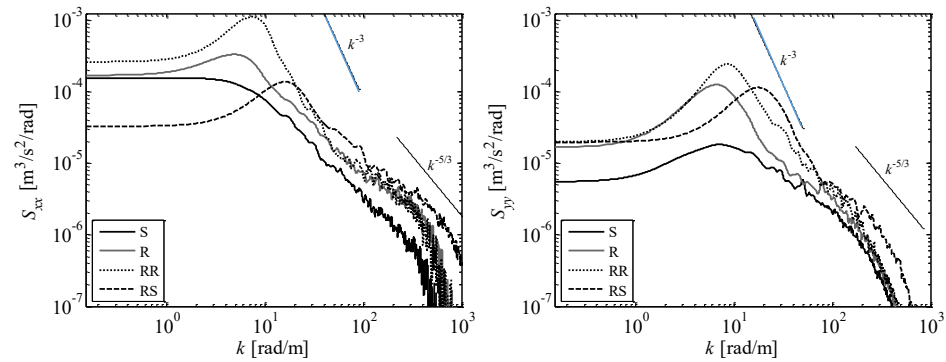

Fig. 7. Autocorrelation (a) and power spectra density (b) in the interface between the main channel and the floodplain, at a fixed downstream distance $x=7.5 \mathrm{~m}$ and at an elevation $z=h_{b}+0.4 h_{f}$.

This is corroborated by the power spectra density for the streamwise fluctuations. In the spanwise direction, only a small peak is observed. Flow cases with synthetic grass in the floodplains (with and without riparian vegetation) feature the modulation of the autocorrelation with a correspondent very pronounced peak in the power spectra density for both streamwise and spanwise velocity fluctuations. These peaks are followed for higher wavenumbers by the slope $k^{-3}$ suggesting the development of large $2 \mathrm{~d}$ structures. The peak of the power spectra is not coincident for all flow cases, suggesting different length scales vortices. Flow case with shrubs in the interface features the smallest length scale structures.

For all flow cases, the universal equilibrium subrange with $k^{-5 / 3}$ slope, typical for $3 \mathrm{~d}$ cascading turbulent flow (e.g. [9]) is observed for higher wavenumbers. For flow cases RR and RS, Fig. 8 presents these functions for points that feature high absolute value of lateral shear stress and at an elevation $z=h b+0.4 h f$.
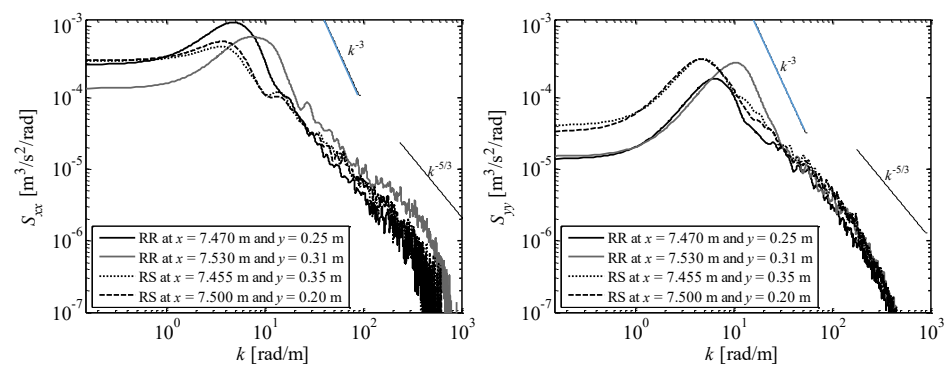

Fig. 8. Power spectra density in the interface between the main channel and the floodplain, at a fixed downstream distance $x=7.5 \mathrm{~m}$ and at an elevation $z=h_{b}+0.4 h_{f}$.

The power spectra density (Fig. 8) shows a clear peak, which is higher for the rod cases in the streamwise direction. The rod cases also present the peak at a higher wavenumber, meaning that vortices are being shed at a higher frequency than for shrub cases.

For the rod cases the turbulent energy is clearly higher in the streamwise than in the transverse direction, while for shrub cases they have approximately the same magnitude. 
Moreover, comparing the results on both sides of the vegetation elements (i.e. main channel $y<0.3 \mathrm{~m}$ and floodplain $y>0.3 \mathrm{~m}$ ) it is clear that shrubs affect equally the flow on both sides, while rods extend their influence in the transverse direction more into the floodplain side than into the main channel.

\section{Conclusions}

In the present paper, the effects of the vegetation in compound channel flows were experimentally evaluated. For each condition, the velocity distribution and the turbulent structure of compound channel flows were characterized.

The results obtained suggest that during the inundation of floodplains, the floodplain and the riparian vegetation have a strong influence on channel conveyance and on the flow structure. The channel conveyance is reduced by the presence of the synthetic grass in the floodplain. That reduction is due not only to floodplains conveyance reduction but also due to the reduction on the main channel conveyance. The riparian vegetation also affects the channel conveyance, but the reduction is almost only due to the reduction of the floodplain conveyance.

The streamwise velocity patterns for flow cases without riparian vegetation are similar. An increase of the velocity gradient between the main channel and the floodplain flows is observed for compound channels with vegetated floodplains. This increase in the velocity gradient results in the increase of the lateral shear stress.

When compared with the case without riparian vegetation, the presence of both rods and shrubs in the interface between the main channel and the floodplain results in a different streamwise velocity pattern. In the main channel, the maximum velocity region moves towards the bottom. The influence of the shrubs is felt in whole main channel.

As proposed by [10] for experiments in single channel, the differences found between the distributions of velocity and lateral shear stress with rods and shrubs in the interface point out the importance of including foliage or equivalent canopy roughness in both flume and numerical experiments.

\section{References}

1. D. W. Knight, K. Shiono, Floodplain Processes (chapter 5), Editors Anderson, M.G., Walling, D.E. and Bates, P.D., John Wiley and Sons Ltd, Chichester, U.K. (1996)

2. J.N. Fernandes, J. B. Leal, A.H. Cardoso, Adv. in Water Resources, 69 (2014)

3. Landcare Notes "Watercourse re-vegetation using indigenous plants". State of Victoria publications (1998)

4. Shiono, K.; Ishigaki, T.; Kawanaka, R. and Heatlie, F., Proceedings of the 33rd Congress of the International Association for Hydraulic Research, Vancouver, Canada (2009)

5. Terrier, B. "Flow characteristics in straight compound channels with vegetation along the main channel", PhD Thesis, Loughborough University (2010)

6. Esfahani, F. and Keshavarzi, A., International Journal of River Basin Management, vol. 8, No. 1(2010)

7. Sun, X. and Shiono, K., Advances in Water Resources, vol. 32 (3), 430-438 (2009)

8. M. Sanjou, I. Nezu, S. Suzuki, K. Itai, J. of Hydrodynamics, 22(5) (2010)

9. Jirka, G., Journal of Hydraulic Research, vol. 39, 2001, No. 6 (2001)

10. Czarnomski, D. Tullos, R. Thomas, A. Simon, Journal of Hydraulic Engineering, 138 (11) (2012) 